Publication List

Juntendo Medical Journal

2014. 60 (5), 492-508

\title{
Publications from Juntendo University Graduate School of Medicine, 2012 [3/6]
}

\section{Department of Neurosurgery}

$<$ Original Articles $>$

1) Esaki T, Harada Y, Nakao Y, Yamamoto T, Mori $\mathrm{K}$ : Unusual case of trigeminal neuralgia caused by remote effect of dermoid cyst in the ipsilateral cerebellar hemisphere. Clin Neurol Neurosurg, doi: 10.1016/j.clineuro.2012.05.010. Epub 2012 May 30.

2) Futakawa S, Nara K, Miyajima M, Kuno A, Ito H, Kaji H, Shirotani K, Honda T, Tohyama Y, Hoshi K, Hanzawa Y, Kitazume S, Imamaki R, Furukawa K, Tasaki K, Arai H, Yuasa T, Abe M, Arai H, Narimatsu H, Hashimoto Y: A unique $\mathrm{N}$-glycan on human transferrin in CSF: a possible biomarker for iNPH. Neurobiol Aging, 2012; 33: 1807-1815.

3) Hayashi A, Nishida M, Seno H, Inoue M, Iwata $\mathrm{H}$, Shirasawa T, Arai H, Kayamori R, Komuro Y, Yanai A: Hemihypoglossal nerve transfer for acute facial paralysis. J Neurosurg, doi: 10.3171/2012.9.JNS1270. Epub 2012 Oct 26.

4) Iimura $Y$, Sugano $H$, Nakajima M, Arai $H$ : Stepwise synchronization through the corpus callosum is one of myclonic jerks. World Neurosurgery, 2012; 77: 399. E5-E8.

5) Kakuda N, Shoji M, Arai H, Furukawa K, Ikeuchi T, Akazawa K, Takami M, Hatsuta H, Murayama S, Hashimoto Y, Miyajima M, Arai H, Nagashima Y, Yamaguchi H, Kuwano R, Nagaike K, Ihara Y; the Japanese Alzheimer's Disease Neuroimaging Initiative: Altered $\gamma$-secretase activity in mild cognitive impairment and Alzheimer's disease. EMBO Mol Med, 2012; 4: 344-352.

6) Kondo A, Date I, Endo S, Fujii K, Fujii Y, Fujimaki T, Hasegawa M, Hatayama T, Hongo $\mathrm{K}$, Inoue $\mathrm{T}$, Ishikawa $\mathrm{M}$, Ito $\mathrm{M}$, Kayama $\mathrm{T}$, Kohmura E, Matsushima T, Munemoto S, Nagahiro S, Ohno K, Okumura T, Ryu H,

An asterisk (*) denotes doctoral works by Japanese students. A dagger $(\dagger)$ denotes doctoral works by non-Japanese students.
Shigeno T, Shirane R, Tagusagawa Y, Tanabe H, Yamada K, Yamakami I: A proposal for standerdized analysis of the results of microvascular decompression for trigeminal neuralgia and hemifacial spasm. Acta Neurochir, 2012; 154: 773-778.

7) Miyajima M, Nakajima M, Ogino I, Miyata $H$, Motoi Y, Arai H: Soluble amyloid precursor protein $\alpha$ in the cerebrospinal fluid as a diagnostic and prognostic biomarker for idiopathic normal pressure hydrocephalus. Eur J Neurol, doi: 10.1111/j.1468-1331.2012.03781. x. Epub 2012 Jun 4.

8) Miyajima M, Shimoji K, Watanabe M, Nakajima M, Ogino I, Arai H: Role of artificial cerebrospinal fluid as perfusate in neuroendoscopic surgery: a basic investigation. Acta Neurochir Suppl, 2012; 113: 103-107.

9) Mori E, Ishikawa M, Kato T, Kazui H, Miyake H, Miyajima M, Nakajima M, Hashimoto M, Kuriyama N, Tokuda T, Ishii K, Kaijima M, Hirata Y, Saito M, Arai H: Guidelines for management of idiopathic normal pressure hydrocephalus: second edition. Neurol Med Chir (Tokyo), 2012; 52: 775-809.

10) Mori K, Yamamoto T, Nakao Y, Miyazaki M, Iwata J, Tamura M, Shiroishi T: Novel neuroprotective effect of cisternal and intracerebral magnesium sul fate solut ion infusion on delayed cerebral death in rat hippocampal neurons after transient global ischemia. Brain Res, 2012; 1480: 72-80.

11) Mori K, Yamamoto T, Miyazaki M, Hara $Y$, Aiko Y, Koike N, Sakamoto S, Nakao Y, Esaki T: Effect of intrathecal magnesium sulfate solution injection via a microcatheter in the cisterna magna on cerebral vasospasm in the canine subarachnoid hemorrhage model. Br J Neurosurg, 2012; 26: 64-68.

12) Nakajima M, Miyajima M, Ogino I, Watanabe M, Hagiwara Y, Segawa T, Kobayashi K, Arai $\mathrm{H}$ : Brain localization of leucine-rich $\alpha 2$-glyco- 
protein and its role. Acta Neurochir Suppl, 2012; 113: 97-101.

*13) Nonaka S, Oishi H, Suga Y, Yamamoto M, Yoshida K, Arai H: Endovascular parent artery occlusion of ruptured vertebral artery dissecting aneurysms. Journal of Neuroendovascular Therapy (JNET), 2012; 6: 98-104.

14) Oishi H, Yamamoto M, Nonaka S, Arai $H$ : Endovascular therapy of internal carotid artery bifurcation aneurysms. J Neurointerv Surg, doi: 10.1136/neurintsurg-2012-010414. Epub 2012 Jul 4.

15) Oishi H, Yamamoto M, Shimizu T, Yoshida K, Arai H: Endovascular therapy of 500 small asymptomatic unruptured aneurysms. AJNR Am J Neuroradiol, 2012; 33: 958-964.

16) Okuma Y, Liu K, Zhang J, Maruo T, Date I, Yoshino T, Ohtsuka A, Otani N, Tomura S, Shima K, Yamamoto Y, Yamamoto H, Takahashi HK, Mori S, Nishibori M: Antihigh mobility group box -1 antibody therapy for traumatic brain injury. Ann Neurol, 2012; 72: 373-384.

17) Shimizu A, Komuro Y, Miyajima M, Arai H: Familial nonsyndromic craniosynostosis with specific deformity of the cranium. J Neurosurg Pediatr, 2012; 10: 560-564.

18) Shimizu Y, Tsutsumi S, Yasumoto Y, Ito M: Carotid cavernous sinus fistula caused by dental implant-associated infection. Am J Otolaryngol, 2012; 33: 352-355.

*19) Tomura S, de Rivero Vaccari JP, Keane RW, Bramlett HM, Dietrich WD: Effects of therapeutic hypothermia on inflammasome signaling after traumatic brain injury. J Cereb Blood Flow Metab, 2012; 32: 1939-1947.

20) Tsutsumi S, Nakamura M, Tabuchi T, Yasumoto $\mathrm{Y}$, Ito $\mathrm{M}$ : An anatomic study of the inferior oblique nerve with highresolution magnetic resonance imaging. Surg Radiol Anat, doi: 10.1007/s00276-012-1040-x. Epub 2012 Nov 6.

21) Tsutsumi S, Nakamura M, Tabuchi T, Yasumoto $\mathrm{Y}$, Ito M: Venous lacunae presenting with unusual upward protrusion: an anatomic study using high-resolution magnetic resonance imaging. Childs Nerv Syst, doi: 10.1007/s00381-0121966-7. Epub 2012 Nov 10.

22) Tsutsumi S, Yasumoto Y, Ito M: Solitary spinal extradural plasmacytoma: a case report and literature review. Clin Neuroradiol, doi: 10.1007/s00062-012-0156-z. Epub 2012 Jun 16.

23) Tsutsumi S, Yasumoto $Y$, Tabuchi T, Ito M: Visualization of the ophthalmic artery by phase-contrast magnetic resonance angiography a pilot study. Surg Radiol Anat, 2012; 34: 833-838.

24) Ueda A, Shimizu A, Natori Y, Sonoue H, Komuro Y, Miyajima M, Arai H: Expression of transforming growth factor- $\beta 1,-\beta 2$, and $-\beta 3$ in plagiocephalic fused and patent coronal suture. J Craniofac Surg, 2012; 23: 755-757.

25) Watanabe M, Miyajima M, Nakajima M, Arai H, Ogino I, Nakamura S, Kunichika M: Expression analysis of high mobility group box -1 protein (HMGB-1) in the cerebral cortex, hippocampus, and cerebellum of the congenital hydrocephalus $(\mathrm{H}-\mathrm{Tx})$ rat. Acta Neurochir Suppl, 2012; 113: 91-96.

26) Watanabe M, Miyajima M, Ogino I, Nakajima M, Arai H: Cerebellar Purkinje cells exhibit increased expression of HMGB-1 and apoptosis in congenital hydrocephalic $\mathrm{H}-\mathrm{Tx}$ rats. Neurosurgery, 2012. doi: 10.1227/NEU.0b013 e31827fcd83. [Epub ahead of print]

27) Yamanaka T, Ishii F, Umemura A, Miyata M, Horiba M, Oka Y, Yamada K, Okita K, Matsukawa N, Ojika K: Temporary deterioration of executive function after subthalamic deep brain stimulation in Parkinson's disease. Clin Neurol Neurosurg, 2012; 114: 347-351.

28) Yatomi K, Yamamoto M, Mitome-Mishima Y, Nonaka S, Yoshida K, Oishi H, Arai H: New experimental model of terminal type aneurysms in Swine: technical note. J Neurol Surg A Cent Eur Neurosurg, 2012; 73: 397-400.

*29) Teranishi K, Scultetus A, Haque A, Stern S, Philbin N, Rice J, Johnson T, Auker C, McCarron R, Freilich D, Arnaud F: Traumatic brain injury and severe uncontrolled haemorrhage with short delay pre-hospital resuscitation in a swine model. Injury, 2012; 43: 585593.

\section{Department of Anatomy}

$<$ Original Articles $>$

1) Hosoyamada Y, Sakai T: Structural arrange- 
ment of collagen fibrils in the periarterial connective tissue of the kidney -- their functional relevance as structural stabilizer against arterial pressure. Anat Sci Int, 2012; 87: 80-87.

* 2) Kumazaki T, Ehara Y, Sakai T: Anatomy and physiology of hamstring injury. Int J Sports Med, 2012; 33: 950-954.

3) Bubenshchikova E, Ichimura K, Fukuyo Y, Powell R, Hsu C, Morrical SO, Sedor JR, Sakai T, Obara T: Wtip and Vangl2 are required for mitotic spindle orientation and cloaca morphogenesis. Biology Open, 2012; 1: 588596.

4) Yamazaki Y, Uka T, Shimizu H, Miyahira A, Sakai T, Marui E: Characteristics of physicians engaged in basic science: A questionnaire survey of physicians in basic science departments of a medical school in Japan. Tohoku J Exp Med, 2012; 228: 75-82.

5) Ichimura K, Bubenshchikova E, Powell R, Fukuyo Y, Nakamura T, Tran U, Oda S, Tanaka M, Wessely O, Kurihara H, Sakai T, Obara T: A comparative analysis of glomerulus development in the pronephros of Medaka and Zebrafish. PLoS One, 2012; 7: e45286.

6) Ichimura K, Fukuyo Y, Nakamura T, Powell R, Sakai T, Obara T: Structural disorganization of pronephric glomerulus in zebrafish mpp5a/ nagie oko mutant. Dev Dyn, 2012; 241: 19221932.

* 7) Kusaba G, Ohsawa I, Ishii M, Inoshita H, Takagi M, Tanifuji C, Takahashi K, Nakamoto J, Yoshida M, Ohi H, Horikoshi S, Kurihara $\mathrm{H}$, Tomino Y: Significance of broad distribution of electron-dense deposits in patients with IgA nephropathy. Med Mol Morphol, 2012; 45: 29-34.

8) Withanage K, Nakagawa K, Ikeda M, Kurihara H, Kudo T, Yang Z, Sakane A, Sasaki T, Hata Y: Expression of RASSF6 in kidney and the implication of RASSF6 and the Hippo pathway in the sorbitol-induced apoptosis in renal proximal tubular epithelial cells. J Biochem, 2012; 152: 111-119.

9) Kajiho Y, Harita Y, Kurihara H, Horita S, Matsunaga A, Tsurumi H, Kanda S, Sugawara N, Miura K, Sekine T, Hattori S, Hattori M, Igarashi T: $\operatorname{SIRP} \alpha$ interacts with nephrin at the podocyte slit diaphragm. FEBS J, 2012;
278: 3010-3021.

$\dagger$ 10) Piao X, Komazawa-Sakon S, Nishina T, Koike M, Piao JH, Ehlken H, Kurihara H, Hara M, Van Rooijen N, Schütz G, Ohmuraya M, Uchiyama Y, Yagita H, Okumura K, He YW, Nakano H: c-FLIP maintains tissue homeostasis by preventing apoptosis and programmed necrosis. Sci Signal, 2012; 5: ra93.

*11) Akutsu H, Sawai T, Sakai T: Morphological analysis of human body expression in the muscle man figures in "Fabrica" of Vesalius. Juntendo Medical Journal, 2012; 58: 151-160.

\section{Department of Organ and Cell Physiology}

$<$ Original Articles >

1) Rawat DK, Hecker P, Watanabe M, Chettimada S, Levy RJ, Okada T, Edwards JG, Gupte SA: Glucose-6-phosphate dehydrogenase and NADPH redox regulates cardiac myocyte L-type calcium channel activity and myocardial contractile function. PLoS One, 2012; 7: e45365.

2) Jin $\mathrm{C}, \mathrm{Wu}$ J, Watanabe M, Okada T, Iesaki T: Mitochondrial $\mathrm{K}+$ channels are involved in ischemic postconditioning in rat hearts. J Physiol Sci, 2012; 62: 325-332.

3) Ichinoseki-Sekine N, Yoshihara T, Kakigi R, Ogura Y, Sugiura T, Naito H: Fiber-type specific expression of $\alpha$-actinin isoforms in rat skeletal muscle. Biochem Biophys Res Commun, 2012; 419: 401-404.

* 4) Hashimoto R, Katoh Y, Nakamura K, Itoh S, Iesaki T, Daida H, Nakazato Y, Okada T: Enhanced accumulation of adipocytes in bone marrow stromal cells in the presence of increased extracellular and intracellular [Ca2+]. Biochem Biophys Res Commun, 2012; 423: 672-678.

$<$ Reviews $>$

1) Naito H, Yoshihara T, Kakigi R, Ichinoseki-Sekine N, Tsuzuki T: Heat stress-induced changes in skeletal muscle: Heat shock proteins and cell signaling transduction. J Phys Fitness Sports Med, 2012; 1: 125-132.

\section{Department of Human Pathology}

$<$ Original Articles >

1) Aritaka N, Ichikawa K, Nakamura H, Yasuda H, Ogura K, Matsumoto T, Komatsu N, Hirano 
T: Attainment of a stringent complete response in multiple myeloma with thalidomide monotherapy. Intern Med, 2012; 51: 27812783.

2) Sato K, Maekawa H, Sakurada M, Orita H, Ito T, Komatsu Y, Hirata F, Wada R: Pathophysiological analysis and strategy for stercoral perforation of the colon. Open Journal Gastroenterology, 2012; 2: 45-50.

3) Ueno H, Mochizuki H, Shirouzu K, Kusumi T, Yamada K, Ikegami M, Kawachi H, Kameoka S, Ohkura Y, Masaki T, Kushima R, Takahashi K, Ajioka Y, Hase K, Ochiai A, Wada R, Iwaya K, Nakamura T, Sugihara K: Study Group for Tumor Deposits without Lymph Nodes Structure in Colorectal Cancer projected by the Japanese Society for Cancer of the Colon and Rectum: Multicentric study for optimal categorization of extramural tumor deposits for colorectal cancer staging. Ann Surg, 2012; 255: 739-746.

4) Ueno H, Mochizuki H, Akagi Y, Kusumi T, Yamada K, Ikegami M, Kawachi H, Kameoka S, Ohkura Y, Masaki T, Kushima R, Takahashi K, Ajioka Y, Hase K, Ochiai A, Wada R, Iwaya K, Shimazaki H, Nakamura T, Sugihara K: Optimal colorectal cancer staging criteria in TNM classification. J Clin Oncol, 2012; 30: 1519-1526.

5) Fujimori $Y$, Fujimori $T$, Imura J, Sugai $T$, Yao T, Wada R, Ajioka Y, Ohkura Y: An assessment of the diagnostic criteria for sessile serrated adenoma/polyps: SSA/Ps using image prodessing software analysis for Ki67 immunohistochemistry. Diagn Pathol, 2012; 29: 59.

6) Sato S, Genda T, Hirano K, Tsuzura H, Narita Y, Kanemitsu Y, Kikuchi T, Iijima K, Wada R, Ichida T: Up-regulated aldo-keto reductase family 1 member B10 in chronic hepatitis C: association with serum alpha-fetoprotein and hepatocellular carcinoma. Liver Int, 2012; 32: 1382-1390.

7) Kimura R, Fujimori T, Ichikawa K, Ajioka Y, Ueno H, Ohkura Y, Kashida H, Togashi K, Yao T, Wada R, Watanabe T, Ochiai A, Sugai T, Sugihara K, Igarashi Y: Desmoplastic reaction in biopsy specimens of early colorectal cancer: A Japanese prospective multicen- ter study. Pathol Int, 2012; 62: 525-531.

* 8) Nakai K, Mitomi H, Alkam Y, Arakawa A, Yao T, Tokuda E, Saito M, Kasumi F: Predictive value of MGMT, hMLH1, hMSH2 and BRCA1 protein expression for pathological complete response to neoadjuvant chemotherapy in basal-like breast cancer patients. Cancer Chemother Pharmacol, 2012; 69: 923-930.

$\dagger$ 9) Imamhasan A, Mitomi H, Saito T, Hayashi T, Takahashi M, Kajiyama Y, Yao T: Immunohistochemical and oncogenetic analyses of the esophageal basaloid squamous cell carcinoma in comparison with conventional squamous cell carcinomas. Hum Pathol, 2012; 43: 20122023.

*10) Nakae K, Mitomi H, Saito T, Takahashi M, Morimoto T, Hidaka Y, Sakamoto N, Yao T, Watanabe S: MUC5AC/ $\beta$-catenin expression and KRAS gene alteration in laterally spreading colorectal tumors. World J Gastroenterol, 2012; 18: 5551-5559.

11) Fukumura $Y$, Takase M, Mitani $K$, Suda $K$, Imamhasan A, Nobukawa B, Ueda A, Abe H, Yao T: Amount of CD4+CD25+ regulatory $\mathrm{T}$ cells in autoimmune pancreatitis and pilonidal sinus. Pancreas, 2012; 41: 910-915.

* 12) Kuroda M, Kuroda K, Arakawa A, Fukumura Y, Kitade M, Kikuchi I, Kumakiri J, Matsuoka S, Brosens IA, Brosens JJ, Takeda S, Yao T: Histological assessment of ompact of ovarian endometrioma and laparoscopic cystectomy on ovarian reserve. J Obstet Gynaecol Res, 2012; 38: 1187-1193.

13) Iwanuma $Y$, Tomita $N$, Amano $T$, Isayama $F$, Tsurumaru M, Hayashi T, Kajiyama Y: Current status of primary malignant melanoma of the esophagus: clinical features, pathology, management and prognosis. J Gastroenterol, 2012; 47: 21-28.

* 14) Nakayama H, Mitomi H, Imamhasan A, Uchida S, Tomita N, Kajiyama Y, Yao T, Watanabe S: Stepwise overexpression of $\mathrm{p} 63$, p53, and cytokeratin 14 during progression of esophageal squamous intraepithelial neoplasia: useful immunohistochemical markers for differential diagnosis. Esophagus, 2012; 9: $1-8$.

15) Iwase A, Onuma E, Nagashima O, Yae T, 
Kunogi M, Hirai S: Long-term survival of adrenal metastasis from non-small cell lung cancer. Int Canc Conf J, 2012; 2: 1-3.

16) Imamhasan A, Mitomi $H$, Saito $T$, Arakawa $A$, Yao T: Clear cell variant of squamous cell carcinoma originating in the esophagus: report of a case with immunohistochemical and oncogenetic analyses: Pathol Int, 2012; 62: 137-143.

17) Nakamura T, Mitomi H, Onozato $W$, Sato $T$, Ikeda A, Naito M, Ogura N, Kamata H, Ooki A, Watanabe M: Laparoscopic resection of a gastrointestinal stromal tumor of the rectum after treatment with imatinib mesylate: report of a case. Surg Today, 2012; 42: 10961099.

18) Kodani T, Osada T, Matsumoto K, Kato J, Higashihara Y, Morimoto T, Ogata C, Taniguchi G, Mizui T, Matsumura Y, Yoshizawa T, Nagahara A, Mitomi H, Yao T, Watanabe S: Endoscopic mucosal resection using a cap-fitted panendoscope as a diagnostic procedure in a case of scirrhous gastric carcinoma. Dig Endosc, 2012; 24: 190.

19) Okubo T, Saito T, Mitomi $H$, Takagi $T$, Torigoe T, Suehara Y, Katagiri H, Murata H, Takahashi M, Ito I, Yao T, Kaneko K: Intraneural lipomatous tumor of the median nerve: Three case reports with a review of literature. Int J Surg Case Rep, 2012; 3: 407411.

20) Miyano G, Hayashi T, Arakawa A, Lane GJ, Okazaki T, Ishizaki Y, Kawasaki S, Yamataka A: Left hepatic lobectomy in a long-term biliary atresia survivor. Afr J Paediatr Surg, 2012; 9: 155-156.

21) Hayashi T, Koike K, Kumasaka T, Saito T, Mitani K, Terao Y, Ogishima D, Yao T, Takeda S, Takahashi K, Seyama K: Uterine angiosarcoma associated with lymphangioleiomyomatosis in a patient with tuberous sclerosis complex: an autopsy case report with immunohistochemical and genetic analysis. Hum Pathol, 2012; 43: 1777-1784.

22) Shida Y, Fujimori T, Tanaka H, Fujimori Y, Kimura R, Ueda H, Ichikawa K, Tomita S, Nagata H, Kubota K, Tsubaki M, Kato H, Yao T, Sugai T, Sugihara K, Ohkura Y, Imura J: Clinicopathological features of serrated adenocarcinoma defined by Mäkinen in dukes' B colorectal carcinoma. Pathobiology, 2012; 79: 169-174.

23) Wakabayashi K, Asanuma K, Takeda Y, Arakawa A, Osawa I, Horikoshi S, Yao T, Tomino Y: Hyaline vascular type of unicentric Castleman's disease with proliferation of glomerular endothelial cells. Clin Nephrol, 2012 Dec 4. [Epub ahead of print]

24) Nakagawa T, Sato K, Moriwaki M, Wada R, Arakawa A, Saito M, Kasumi F: Successful endocrine therapy for locally advanced mucinous carcinoma of the breast. Bresast J, 2012; 18: $632-633$.

25) Yokoi H, Arakawa A, Inoshita A, Ikeda K: Novel use of a Weerda laryngoscope for transoral excision of a cervical ganglioneuroma: a case report. J Med Case Rep, 2012; 6: 88.

*26) Tokuda E, Seino Y, Arakawa A, Saito M, Kasumi F, Hayashi S, Yamaguchi Y: Estrogen receptor- $\alpha$ directly regulates sensitivity to paclitaxel in neoadjuvant chemotherapy for breast cancer. Breast Cancer Res Treat, 2012; 133: 427-436.

*27) Takahashi Y, Iwai M, Kawai T, Arakawa A, Ito T, Sakurai-Yageta M, Ito A, Goto A, Saito M, Kasumi F, Murakami Y: Aberrant expression of tumor suppressors CADM1 and 4. 1B in invasive lesions of primary breast cancer. Breast Cancer, 2012; 19: 242-252.

28) Yonezawa I, Saito T, Nakahara D, Won J, Wada T, Kaneko K: Synovial sarcoma of the cauda equina. J Neurosurg Spine, 2012; 16: 187-190.

29) Kikuta K, Kubota D, Saito T, Orita H, Yoshida A, Tsuda H, Suehara Y, Katai H, Shimada Y, Toyama Y, Sato K, Yao T, Kaneko K, Beppu Y, Murakami Y, Kawai A, Kondo T: Clinical proteomics identified ATP-dependent RNA helicase DDX39 as a novel biomarker to predict poor prognosis of patients with gastrointestinal stromal tumor. J Proteomics, 2012; 75: 1089-1098.

30) Kubota D, Okubo T, Saito T, Suehara Y, Orita H, Yoshida A, Tsuda H, Kikuta K, Gotoh M, Katai H, Shimada Y, Yao T, Kaneko K, Kawai A, Kondo T: Validation study on pfetin and ATP-dependent RNA helicase DDX39 as prognostic biomarkers in gastrointestinal stromal 
tumor. Jpn J Clin Oncol, 2012; 42: 730-741.

31) Saito T, Mitomi H, Torigoe $T$, Takagi $T$, Suehara Y, Okubo T, Kaneko K, Yao T: Malignant granular cell tumor with unusual long clinical course. An autopsy case with review of literatures. J Cancer Sci Ther, 2012; 4: 260-263.

32) Saito T, Yokotsuka M, Motoi T, Iwasaki H, Nagao T, Ladanyi M, Yao T: EWS-WT1 chimeric protein in desmoplastic small round cell tumor is a potent transactivator of FGFR4. J Cancer Sci Ther, 2012; 4: 335-340.

33) Ho AL, Vasudeva SD, Laé M, Saito T, Barbashina V, Antonescu CR, Ladanyi M, Schwartz GK: PDGF receptor alpha is an alternative mediator of rapamycin-induced Akt activation: implications for combination targeted therapy of synovial sarcoma. Cancer Res, 2012; 72: 4515-4525.

\section{Department of Cellular and Molecular Pharmacology}

$<$ Original Articles $>$

1) Suzuki T, Shioya T, Murayama T, Sugihara M, Odagiri F, Nakazato Y, Nishizawa H, Chugun A, Sakurai T, Daida H, Morimoto S, Kurebayashi N: Multistep ion channel remodeling and lethal arrhythmia precede heart failure in a mouse model of inherited dilated cardiomyopathy. PLoS One, 2012; 7: e35353.

2) Kakizawa S, Yamazawa T, Chen Y, Ito A, Murayama T, Oyamada H, Kurebayashi N, Sato O, Watanabe M, Mori N, Oguchi K, Sakurai T, Takeshima H, Saito N, Iino M: Nitric oxide-induced calcium release via ryanodine receptors regulates neuronal function. EMBO J, 2012; 31: 417-428.

3) Ichikawa-Tomikawa N, Ogawa J, Douet V, Xu Z, Kamikubo Y, Sakurai T, Kohsaka S, Chiba H, Hattori N, Yamada Y, ArikawaHirasawa E: Laminin $\alpha 1$ is essential for mouse cerebellar development. Matrix Biology, 2012; 31: 17-28.

\section{Department of Gastroenterology}

$<$ Original Articles $>$

1) Harada K, Hirohara J, Ueno Y, Nakano T, Kakuda Y, Tsubouchi H, Ichida T, Nakanuma Y: Incidence of and risk factors for hepatocel- lular carcinoma in primary biliary cirrhosis: national data from Japan. Hepatology, 2012 Nov 29. doi: 10.1002/hep.26176. [Epub ahead of print]

2) Hasegawa K, Kokudo N, Makuuchi M, Izumi N, Ichida T, Kudo M, Ku Y, Sakamoto M, Nakashima O, Matsui O, Matsuyama Y: Comparison of resection and ablation for hepatocellular carcinoma: a cohort study based on a Japanese nationwide survey. J Hepatol, doi: 10.1016/j.jhep.2012.11.009. Epub 2012 Nov 21.

3) Yamamoto K, Miyake Y, Ohira H, Suzuki Y, Zeniya M, Onji M, Tsubouchi H; Hiroishi K, Yoshizawa K, Morizane T, Hibi T, Aoyagi Y, Nakanuma Y, Hirohara J, Takikawa H, Ishibashi H, Shimoda S, Sakisaka S, Nakamuta M, Matsuzaki Y, Saibara T, Ueno Y, Miyakawa H, Kokudo N, Egawa H, Maehara Y, Mochida S, Sakaida I, Fujisawa T, Suzuki K, Inoue K, Ichida T, Yokosuka O, Fukui H, Moriwaki H, Mori M, Mori T, Nagino M, Sata N, Tazuma S, Yasaka T, Tsuyuguchi T, Shoda J, Honda M, Yamaue H, Unno M, Hayashi N: Prognosis of autoimmune hepatitis showing acute presentation. Hepatol Res, doi: 10.1111/j.1872-034X. 2012.01109.x. Epub 2012 Oct 22.

4) Nakamura M, Nishida N, Kawashima M, Aiba Y, Tanaka A, Yasunami M, Nakamura H, Komori A, Nakamuta M, Zeniya M, Hashimoto E, Ohira H, Yamamoto K, Onji M, Kaneko S, Honda M, Yamagiwa S, Nakao K, Ichida T, Takikawa H, Seike M, Umemura T, Ueno Y, Sakisaka S, Kikuchi K, Ebinuma H, Yamashiki N, Tamura S, Sugawara Y, Mori A, Yagi S, Shirabe K, Taketomi A, Arai K, Monoe K, Ichikawa T, Taniai M, Miyake Y, Kumagi T, Abe M, Yoshizawa K, Joshita S, Shimoda S, Honda K, Takahashi H, Hirano K, Takeyama Y, Harada K, Migita K, Ito M, Yatsuhashi H, Fukushima N, Ota H, Komatsu T, Saoshiro T, Ishida J, Kouno H, Kouno H, Yagura M, Kobayashi M, Muro T, Masaki N, Hirata K, Watanabe Y, Nakamura Y, Shimada M, Hirashima N, Komeda T, Sugi K, Koga M, Ario K, Takesaki E, Maehara Y, Uemoto S, Kokudo N, Tsubouchi H, Mizokami M, Nakanuma Y, Tokunaga K, Ishibashi H: Genome-wide association study identifies TNFSF15 and POU2AF1 as susceptibility 
loci for primary biliary cirrhosis in the Japanese population. Am J Hum Genet, 2012; 91: 721-728.

5) Marubashi S, Umeshita K, Asahara T, Fujiwara K, Haga H, Hashimoto T, Hatakeyama $\mathrm{K}$, Ichida $\mathrm{T}$, Kanematsu T, Kitajima M, Kiyosawa K, Makuuchi M, Miyagawa S, Satomi S, Soejima Y, Takada Y, Tanaka N, Teraoka S, Monden M: Steroid-free living donor liver transplantation for $\mathrm{HCV}^{--} \mathrm{a}$ multicenter prospective cohort study in Japan. Clin Transplant, 2012; 26: 857-867.

6) Sakurai K, Nagahara A, Inoue K, Akiyama J, Mabe K, Suzuki J, Habu Y, Araki A, Suzuki T, Satoh K, Nagami H, Harada R, Tano N, Kusaka M, Fujioka Y, Fujimura T, Shigeto N, Oumi T, Miwa J, Miwa H, Fujimoto K, Kinoshita Y, Haruma K: Efficacy of omeprazole, famotidine, mosapride and teprenone in patients with upper gastorointestinal symptoms: an omeprazole-controlled randomized study (J-FOCUS). BMC Gastroenterol, 2012; 12: 42 .

7) Nagahara A, Hojo M, Asaoka D, Sasaki H, Oguro M, Mori H, Matsumoto K, Osada T, Yoshizawa T, Watanabe S: Clinical feature of asymptomatic reflux esophagitis in patients who underwent upper gastrointestinal endoscopy. J Gastroenterol Hepatol, 2012; 3: S53-S57.

8) Hojo M, Takahashi T, Nagahara A, Sasaki H, Oguro M, Asaoka D, Watanabe S: Analysis of brain activity during visceral stimulation. J Gastroenterol Hepatol, 2012; 3: S49-S52.

9) Hotta K, Saito Y, Fujihiro M, Ikehara H, Ikematsu H, Kobayashi N, Sakamoto N, Takeuchi Y, Uraoka T, Yamaguchi Y: Impact of endoscopic submucosal dissection for the therapeutic strategy of large colorectal tumors. J Gastroenterol Hepatol, 2012; 27: 510515.

10) Shibuya T, Osada T, Nomura O, Fukuo Y, Watanabe S: The origin of hyperamylasemia associated with peroral double-balloon endoscopy. J Clin Gastroenterol, 2012; 46: 888889.

11) Shibuya T, Mori H, Takeda T, Konishi M, Fukuo Y, Matsumoto K, Beppu K, Sakamoto N, Osada T, Nagahara A, Otaka M, Ogihara T,
Watanabe S: The relationship between physical activity level and completion rate of small bowel examination in patients undergoing capsule endoscopy. Intern Med, 2012; 51: 997-1001.

12) Kato J, Nagahara A, Kodani $T$, Higashihara $Y$, Matsumura Y, Osada T, Yoshizawa T, Suyama M, Watanabe S: Phase I clinical trial of peptide vaccination with KIF20A and VEGFR1 epitope peptides in patients with advanced pancreatic cancer. Pancreatic Dis Ther, 2012; 2: 102.

13) Corridoni D, Pastorelli L, Mattioli B, Locovei S, Ishikawa D, Arseneau KO, Pizarro TT: Prbiotic bacteria regulate intestinal epithelial permeability in experimental ileitis by a TNF-dependent mechanism. PLoS One, 2012; 7: e420 670.

* 14) Nakae K, Mitomi H, Saito T, Takahashi M, Morimoto T, Hidaka Y, Sakamoto N, Yao T, Watanabe S: MUC5AC/ $\beta$-catenin expression and KRAS gene alteration in laterally spreading colorectal tumors. World J Gastroenterol, 2012; 18: 5551-5559.

*15) Igusa Y, Yamashina S, Izumi K, Inami Y, Fukada H, Komatsu M, Tanaka K, Ikejima K, Watanabe S: Loss of autophagy promotes murine acetaminophen hepatotoxicity. J Gastroenterol, 2012; 47: 433-443.

$\dagger 16$ ) Lee SY, Tomoyoshi S, Haga K, Sasaki H, Ogata C, Nomura O, Fukuo Y, Abe W, Osada $T$, Nagahara A, Ogihara T, Kamiyama H, Sakamoto K, Watanabe S: Multiple carcinoid tumors of the small intestine preoperatively diagnosed by double-balloon endoscopy. Med Sci Monit, 2012; 18: CS109-CS112.

17) Kodani $T$, Osada T, Matsumoto K, Kato J, Higashihara Y, Morimoto T, Ogata C, Taniguchi G, Mizui T, Matsumura Y, Yoshizawa T, Nagahara A, Mitomi H, Yao T, Watanabe S: Endoscopic mucosal resection using a cap-fitted panendoscope as a diagnostic procedure in a case of scirrhous gastric carcinoma. Digestive Endoscopy, 2012; 24: 190.

18) Tamaki K, Otaka M, Shibuya T, Sakamoto N, Yamamoto S, Odashima M, Itoh $\mathrm{H}$, Watanabe S: Traditional Herbal Medicine, Rikkunshito, Induces HSP60 and Enhances Cytoprotection of Small Intestinal Mucosal Cells as a Non- 
toxic Chaperone Inducer. Evid Based Complement Alternat Med, 2012; 2012: 278958.

* 19) Fukada H, Yamashina S, Izumi K, Komatsu M, Tanaka K, Ikejima K, Watanabe S: Suppression of autophagy sensitizes Kupffer cells to endotoxin. Hepatol Res, 2012; 42: 1112-1118.

*20) Saito H, Ito K, Sugiyama M, Matsui T, Aoki Y, Imamura M, Murata K, Masaki N, Nomura $\mathrm{H}$, Adachi H, Higa S, Enomoto N, Sakamoto N, Kurosaki M, Mizokami M, Watanabe S: Factors responsible for the discrepancy between IL28B polymorphism prediction and the viral response to peginterferon plus ribavirin therapy in Japanese chronic hepatitis C patients. Hepatol Res, 2012; 42: 958-965.

21) Sato S, Genda T, Hirano K, Tsuzura H, Narita Y, Kanemitsu Y, Kikuchi T, Iijima K, Wada R, Ichida $\mathrm{T}$ : Up-regulated aldo-keto reductase family 1 member B10 in chronic hepatitis C: association with serum alpha-fetoprotein and hepatocellular carcinoma. Liver Int, 2012; 32: 1382-1390.

22) Konuma H, Fu K, Konuma I, Ueyama H, Takahashi T, Ogura K, Miyazaki A, Watanabe S: Endoscopic full-thickness resection of a lateral spreading rectal tumor after unplanned injection of dilute hyaluronic acid into the subserosal layer (with video). Tech Coloproctol, 2012; 16: 247-250.

23) Monden M, Sakon M, Sakata Y, Ueda Y, Hashimura E; FAIT Research Group: 5-fluorouracil arterial infusion + interferon therapy for highly advanced hepatocellular carcinoma: A multicenter, randomized, phase II study. Hepatol Res, 2012; 42: 150-165.

24) Iizuka M, Nakagomi O, Nanjo H, Chiba M, Fukushima T, Sugita A, Sagara S, Horie Y, Watanabe S: Molecular cloning reveals nearly a half of patients with Crohn's disease have an antibody to peroxiredoxin 6 -like protein. J Gastroenterol Hepatol, 2012; 27: 1388-1394.

25) $\mathrm{Fu} \mathrm{L}$, Kitamura $\mathrm{T}$, Iwabuchi $\mathrm{K}$, Ichinose $\mathrm{S}$, Yanagida M, Ogawa H, Watanabe S, Maruyama T, Suyama M, Takamori K: Sphingosine kinase 1 regulates migration of sinusoidal endothelial cells isolated from regenerating liver in rats. World J Gastroenterol, 2012; 48: 5034-5041.

26) Kawaguchi T, Sumida Y, Umemura A, Matsuo
K, Takahashi M, Takamura T, Yasui K, Saibara T, Hashimoto E, Kawanaka M, Watanabe S, Kawata S, Imai Y, Kokubo M, Shima T, Park H, Tanaka H, Tajima K, Yamada R, Matsuda F, Okanoue T; Japan Study Group of Nonalcoholic Fatty Liver Diease (JSG-NAFLD) : Genetic polymorphisms of the human PNPLA3 gene are strongly associated with severity of non-alcoholic fatty liver disease in Japanese. PLoS One, 2012; 7: e38322.

27) Imajo K, Fujita K, Yoneda M, Nozaki Y, Ogawa Y, Shinohara Y, Kato S, Mawatari H, Shibata W, Kitani H, Ikejima K, Kirikoshi H, Nakajima N, Saito S, Maeyama S, Watanabe S, Wada K, Nakajima A: Hyperresponsivity to low-dose endotoxin during progression to nonalcoholic steatohepatitis is regulated by leptin-mediated signaling. Cell Metab, 2012; 16: 44-54.

*28) Kikuchi D, Iizuka T, Hoteya S, Yamada A, Furuhata T, Yamashita S, Domon K, Nakamura M, Matsui A, Mitani T, Ogawa O, Watanabe S, Yahagi N, Kaise M: Safety and efficacy of secondary endoscopic submucosal dissection for residual gastric carcinoma after primary endoscopic submucosal dissection. Digestion, 2012; 86: 288-293.

* 29) Nakayama H, Mitomi H, Imamhasan A, Uchida S, Tomita N, Kajiyama Y, Yao T, Watanabe S: Stepwise overexpression of $\mathrm{p} 63$, p53, and cytokeratin 14 during progression of esophageal squamous intraepithelial neoplasia: useful immunohistochemical markers for differential diagnosis. Esophagus, 2012; 9: 1-8.

$\dagger 30$ ) Abudoxueke M, Yamashina S, Ikejima K, Komatsu M, Tanaka K, Watanabe S: Loss of autophagy enhances diethylnitrosamin-induced liver injury. Juntendo Medical Journal, 2012; 319-324.

$<$ Books $>$

1) Ichida T, Matsuda Y: Hepatocellular carcinoma-Etiology, Risk Factors and Prevention. Encyclopedia of Cancer. Edited by Schwab M, Springer Berlin Heidelberg, 2012: 1673-1677.

\section{Department of Cardiovascular Medicine}

$<$ Original Articles >

1) Okai I, Inoue K, Maruyama M, Maruyama $S$, Komatsu K, Nishizawa H, Okazaki S, Fuji- 
wara Y, Sumiyoshi M, Daida H: Transbrachial intra-aortic balloon pumping for a patient with fulminant myocarditis. Heart Vessels, 2012; 27: 639-642.

2) Daigo K, Yamaguchi N, Kawamura T, Matsubara K, Jiang S, Ohashi R, Sudou Y, Kodama T, Naito M, Inoue K, Hamakubo T: The proteomic profile of circulating pentraxin 3 (PTX3) complex in sepsis demonstrates the interaction with azurocidin 1 and other components of neutrophil extracellular traps. Mol Cell Proteomics, 2012; 11: M111. 015073.

3) Tamura Y, Ono T, Kuwana M, Inoue K, Takei M, Yamamoto T, Kawakami T, Fujita J, Kataoka M, Kimura K, Sano M, Daida H, Satoh T, Fukuda K: Human pentraxin 3 (PTX3) as a novel biomarker for the diagnosis of pulmonary arterial hypertension. PLoS One, 2012; 7: e45834.

4) Komatsu T, Onda T, Murayama G, Yamanouchi M, Inukai M, Sakai A, Kikuta M, Branch J, Aoki M, Tierney LM Jr, Inoue K: Predicting bacteremia based on nurse-assessed food consumption at the time of blood culture. J Hosp Med, 2012; 7: 702-705.

5) Morita H, Fujimoto S, Kondo $T$, Arai $T$, Sekine T, Matsutani H, Sano T, Kondo M, Kodama T, Takase S, Narula J: Prevalence of computed tomographic angiography-verified high risk plaques and significant luminal stenosis in patients with zero coronary calcium score. Int J Cardiol, 2012; 58: 272278.

6) Kodama T, Kondo T, Oida A, Fujimoto S, Narula J: Computed tomographic angiography-verified plaque characteristics and slowflow phenomenon during percutaneous coronary intervention. JACC Cardiovasc Interv, 2012; 5: 636-643.

7) Fujimoto S, Kondo T, Kodama T, Orihara T, Sugiyama J, Kondo M, Endo A, Fukazawa H, Nagaoka H, Oida A, Ikeda T, Yamazaki J, Takase S, Narula J: Coronary computed tomography angiography-based coronary risk stratification in subjects presenting with no or atypical symptoms. Circ J, 2012; 76: 2419-2425.

8) Fujimoto S, Kondo T, Kodama T, Takase S, Narula J: Delayed plaque enhancement by coronary $\mathrm{CT}$ angiography. JACC Cardiovasc Imaging, 2012; 5: 1181-1182.

9) Konishi H, Tokano T, Nakazato Y, Komatsu S, Suwa S, Komatsu K, Hayashi H, Sekita G, Sumiyoshi M, Bito F, Kizu K, Daida H: Twiddler's syndrome detected by patient's complaints of implantable cradioveretr-defibrillator rotation in the subcutaneous pocket. Journal of Arrhythmia, 2012; 28: 239-241.

10) Matsumori R, Miyazaki T, Shimada K, Kume A, Kitamura Y, Oshida K, Yanagisawa N, Kiyanagi T, Hiki M, Fukao K, Hirose K, Ohsaka H, Mokuno H, Daida H: High levels of very long-chain saturated fatty acid in erythrocytes correlates with atherogenic lipoprotein profiles in subjects with metabolic syndrome. Diabetes Res Clin Pract, doi: 10. 1016/j. diabres. 2012.10.025. Epub 2012 Nov 10.

* 11) Matsumori R, Shimada K, Kiyanagi T, Hiki M, Fukao K, Hirose K, Ohsaka H, Miyazaki T, Kume A, Yamada A, Takagi A, Ohmura H, Miyauchi K, Daida H: Clinical significance of the measurements of urinary liver-type fatty acid binding protein levels in patients with acute coronary syndrome. J Cardiol, 2012; 60: 168-173.

12) Suzuki T, Shioya $T$, Murayama $T$, Sugihara M, Odagiri F, Nakazato Y, Nishizawa H, Chugun A, Sakurai T, Daida H, Morimoto S, Kurebayashi N: Multistep ion channel remodeling and lethal arrhythmia precede heart failure in a mouse model of inherited dilated cardiomyopathy. PLoS One, 2012; 7: e35353.

*13) Yamase M, Nakazato Y, Daida H: Effectiveness of amiodarone versus bepridil in achieving conversion to sinus rhythm in patients with persisitent atrial fibrillation: a randomized trial. Heart, 2012; 98: 1067-1071.

*14) Miura S, Sumiyoshi M, Tsuchiya H, Maruyama M, Seigen I, Okai I, Masaki Y, Okazaki S, Inoue K, Fujiwara Y, Komatsu K, Hayashi H, Sekita G, Tokano T, Nakazato Y, Daida H: The use of serum bepridil concentration as a safe rhythm control strategy in patients with atrial tachyarrhythmias. J Arrhythmia, 2012; 28: 187-191.

*15) Hashimoto R, Katoh Y, Nakamura K, Itoh S, 
Iesaki T, Daida H, Nakazato Y, Okada T: Enhanced accumulation of adipocytes in bone marrow stromal cells in the presence of increased extracellular and intracellular $\left[\mathrm{Ca}^{2+}\right]$. Biochem Biophys Res Commun, 2012; 423: 672-678.

* 16) Hirano K, Yamashita T, Suzuki S, Hayama E, Matsuoka J, Otsuka T, Sagara K, Fu LT, Sawada H, Aizawa T, Nakazato Y, Daida H: Relationship between 24-h Holter recordings and clinical outcomes in patients with permanent atrial fibrillation. J Cardiol, 2012; 60: $42-46$.

17) Tokano T, Nakazato $Y$, Komatsu K, Suzuki T, Hayashi H, Sekita G, Kawano Y, Nakazato K, Sumiyoshi M, Daida H: Effective refractory periods in the right atrium in patients with atrioventricular nodal reentrant tachycardia. J HK Coll Cardiol, 2012; 20: 31-37.

18) Matsuzaka T, Atsumi A, Matsumori R, Nie T, Shinozaki H, Suzuki-Kemuriyama N, Kuba M, Nakagawa Y, Ishii K, Shimada M, Kobayashi K, Yatoh S, Takahashi A, Takekoshi K, Sone H, Yahagi N, Suzuki H, Murata S, Nakamuta M, Yamada N, Shimano H: Elovl6 promotes nonalcoholic steatohepatitis. Hepatology, 2012; 56: 2199-208.

* 19) Nishizaki Y, Yamagami S, Haga K, Sesoko M, Yamashita H, Miyauchi K, Daida H: Usefulness of prominently projected aortic arch on chest radiograph to predict severe tortuosity of the right subclavian or brachiocephalic artery in patients aged $>44$ years undergoing coronary angiography with a right radial artery approach. Am J Cardiol, 2012; 110: 203-207.

20) Nishizaki Y, Yamagami S, Suzuki H, Joki Y, Takahashi S, Sesoko M, Yamashita H, Kuremoto K, Shinozaki T, Daida H: Red blood cell distribution width as an effective tool for detecting fatal heart failure in super-elderly patients. Intern Med, 2012; 51: 2271-2276.

*21) Yamada A, Takeuchi Y, Nishizaki Y, Daida H: Bag-valve-mask ventilation with airway adjuncts improves neurological outcomes of in-hospital cardiac arrest. Intern Med, 2012; 51: 1517-1521.

*22) Ohsaka H, Shimada K, Fukao K, Sai E, Fukushima Y, Matsuda H, Hirose K, Yoshi- hara T, Matsumori R, Hiki M, Kiyanagi T, Kume A, Yamashita H, Miyazaki T, Daida H: Clinical and prognostic features in asymptomatic and symptomatic patients with arteriosclerosis obliterans. World Journal of Cardiovascular Diseases, 2012; 2: 1-7.

$\dagger 23$ ) Chiang S, Daimon M, Ishii K, Miyazaki S, Koiso Y, Suzuki H, Miyauchi K, Yang B, Yeh M, Hwang B, Daida H: A novel global strain diastolic index correlates with Plasma NT-pro BNP levels in asymptomatic hypertensive patients with preserved left ventricular ejection fraction. Journal of Echocardiography, 2012; 10: 56-64.

*24) Kinoshita R, Matsunaga E, Nishizaki Y, Yokoyama T, Itou S, Miyauchi K, Daida H: Coronary Artery Calcium Score as a Predictor of Coronary Stenosis in Patients with Diabetes Mellitus. J Jpn Coron Assoc, 2012; 18: $122-129$.

*25) Suzuki T, Nakazato Y, Nishizawa H, Chugun A, Murayama T, Sakurai T, Daida H, Morimoto S, Kurebayashi N: Arrhythmogenic activity in left ventricles of dilated cardiomyopathy (DCM) model mice -Difference between adrenergic and cholinergic effects-. Juntendo Medical Journal, 2012; 58: 44-48.

$<$ Reviews $>$

1) Inoue K, Kodama T, Daida H: Pentraxin 3: a novel biomarker for inflammatory cardiovascular disease. Int J Vasc Med, 2012; 2012: 657025.

2) Fujimoto S, Kondo T, Narula J: Evaluation of plaque morphology by coronary $\mathrm{CT}$ angiography. Cardiol Clin, 2012; 30: 69-75.

\section{Department of Respiratory Medicine}

$<$ Original Articles >

1) Ando K, Doi T, Moody SY, Ohkuni Y, Sato S, Kaneko N: The effect of comorbidity on the prognosis of acute lung injury and acute respiratory distress syndrome. Intern Med, 2012; 51: 1835-1840.

2) Ando K, Takahashi F, Motojima S, Nakashima K, Kaneko N, Hoshi K, Takahashi K: Possible role of tocilizumab, anti-interleukin 6 receptor antibody, the new approach to cancer cachexia. J Clin Oncol, 2012; 31: e69e72. 
3) Ando K, Tobino K, Kurihara M, Kataoka H, Doi T, Hoshika Y, Takahashi K, Seyama K: Quantitative CT analysis of small pulmonary vessels in Lymphangioleiomyomatosis. Eur J Radiol, 2012; 81: 3925-3930.

4) Fujii M, Iwakami SI, Takagi H, Itoigawa $Y$, Ichikawa M, Iwakami N, Ishiwata T, Seyama $\mathrm{K}$, Takahashi K: Factors influencing weaning from mechanical ventilation in elderly patients with severe pneumonia. Geriatr Gerontol Int, 2012; 12: 277-283.

5) Hayashi T, Koike K, Kumasaka T, Saito T, Mitani K, Terao Y, Ogishima D, Yao T, Takeda S, Takahashi K, Seyama K: Uterine angiosarcoma associated with lymphangioleiomyomatosis in a patient with tuberous sclerosis complex: an autopsy case report with immunohistochemical and genetic analysis. Hum Pathol, 2012; 43: 1777-1784.

6) Ishiwata T, Harada N, Ko R, Hara M, Sekiya M, Sasaki M, Nobukawa B, Takahashi K: Malignant lymphoma with diffuse cardiac involvement detected by multiple imaging examinations: a case report. J Med Case Rep, 2012; 6: 193.

7) Kaira K, Endo M, Shukuya T, Kenmotsu H, Naito T, Ono A, Tsuya A, Nakamua Y, Takahashi T, Murakami H, Kondo H, Nakajima T, Yamamoto N: ${ }^{18} \mathrm{~F}-\mathrm{FDG}$ uptake on PET could be a predictive marker of excision repair cross-complementation group 1 (ERCC1) expression in patients with thoracic neoplasms? Neoplasma, 2012; 59: 257-263.

8) Koyama R, Miura K, Yae S, Murakami A, Takahashi K: Effect of continuation of chemotherapy on quality of life in elderly patients with lung cancer -Comparison with that in younger patients- ${ }^{-}$. Juntendo Medical Journal, 2012; 58: 506-511.

* 9) Makino F, Ito J, Abe Y, Harada N, Kamachi F, Yagita H, Takahashi K, Okumura K, Akiba H: Blockade of CD70-CD27 iponteraction inhabits induction of allergic lung inflammation in mice. Am J Respir Cell Mol Biol, 2012; 47: 298-305.

* 10) Minakata K, Takahashi F, Nara T, Hashimoto M, Tajima K, Murakami A, Nurwidya F, Yae S, Koizumi F, Moriyama H, Seyama K, Nishio $\mathrm{K}$, Takahashi K: Hypoxia induces gefitinib resistance in non-small-cell lung cancer with both mutant and wild-type epidermal growth factor receptors. Cancer Sci, 2012; 103: 19461954.

*11) Muraki K, Koyama R, Honma Y, Yagishita S, Shukuya T, Ohashi R, Takahashi F, Kido K, Iwakami S, Sasaki S, Iwase A, Takahashi K: Hydration with magnesium and mannitol without furosemide prevents the nephrotoxicity induced by cisplatin and pemetrexed in patients with advanced non-small cell lung cancer. J Thorac Dis, 2012; 4: 562-568.

12) Nagashima O, Iwase A, Yae T, Kadoya K, Ishimori A, Kuriyama S, Koyama R, Homma $\mathrm{N}$, Ienaga $\mathrm{H}$, Takahashi $\mathrm{K}$ : A case of pseudomembranous aspergillus tracheobronchitis in an asthma patient receiving inhaled corticosteroid therapy. The Journal of the Japan Society for Respiratory Endoscopy, 2012; 34: 71-73.

13) Nagashima O, Ohashi R, Yoshioka Y, Inagaki A, Tajima M, Koinuma Y, Iwakami S, Iwase A, Sasaki S, Tominaga S, Takahashi K: High prevalence of gene abnormalities in young patients with lung cancer. J Thorac Dis, 2012; 5: 27-30.

14) Nagashima O, Suzuki $Y$, Iwase A, Takahashi $\mathrm{K}$ : Acute hemorrhage in a giant bulla. Intern Med, 2012; 51: 2673.

15) Shukuya T, Takahashi T, Harada H, Akamatsu H, Sakaguchi C, Imai H, Ono A, Nakamura Y, Tsuya A, Kenmotsu H, Naito T, Murakami $\mathrm{H}$, Endo M, Takahashi K, Yamamoto N: Comparison of vinorelbine plus cisplatin and $\mathrm{s}^{-1}$ plus cisplatin in concurrent chemoradiotherapeutic regimens for unresectable stage III non-small cell lung cancer. Anticancer Res, 2012; 32: 675-680.

*16) Yae S, Takahashi F, Yae T, Yamaguchi T, Tsukada R, Koike K, Minakata K, Murakami A, Nurwidya F, Kato M, Tamada M, Yoshikawa M, Kobayashi H, Seyama K, Takahashi K: Hochuekkito (TJ-41), a Kampo Formula, Ameliorates Cachexia Induced by Colon 26 Adenocarcinoma in Mice. Evid Based Complement Alternat Med, 2012; 2012: 976926.

17) Yae $T$, Tsuchihashi $K$, Ishimoto $T$, Motohara T, Yoshikawa M, Yoshida GJ, Wada T, Masuko T, Mogushi K, Tanaka H, Osawa T, Kanki Y, 
Minami T, Aburatani H, Ohmura M, Kubo A, Suematsu M, Takahashi K, Saya H, Nagano O: Alternative splicing of CD44 mRNA by ESRP1 enhances lung colonization of metastatic cancer cell. Nat Commun, 2012; 3: 883.

* 18) Yoshimi K, Ueki J, Seyama K, Takizawa M, Yamaguchi S, Kitahara E, Fukazawa S, Takahama Y, Ichikawa M, Takahashi K, Fukuchi Y: Pulmonary rehabilitation program including respiratory conditioning for chronic obstructive pulmonary disease (COPD) : Improved hyperinflation and expiratory flow during tidal breathing. J Thorac Dis, 2012; 4: 259-264.

* 19) Tobino K, Hirai T, Johkoh T, Kurihara M, Fujimoto K, Tomiyama N, Mishima M, Takahashi K, Seyama K: Differentiation between Birt-Hogg-Dubé syndrome and lymphangioleiomyomatosis: quantitative analysis of pulmonary cysts on computed tomography of the chest in 66 females. Eur J Radiol, 2012; 81: 1340-1346.

*20) 佐藤由起子, 深澤伸慈, 熱田了, 武田 康一, 鈴木 勉 : 高頻度振動換気方法 (HFOV) 施行 時の加温加湿不良に対する改良回路の検討. 日本呼吸ケア・リハビリテーション学会誌, 2012; 22: 198-203.

$<$ Reviews $>$

1) Nurwidya F, Murakami A, Takahashi F, Takahashi K: Lung cancer stem cells: tumor biology and clinical implications. Asia Pac J Clin Oncol, 2012; 8: 217-222.

2) Nurwidya F, Murakami A, Takahashi F, Takahashi K: Molecular mechanisms contributing to resistance to tyrosine kinase-targeted therapy for non-small cell lung cancer. Cancer Bio Med, 2012; 9: 18-22.

3) Nurwidya F, Takahashi F, Minakata K, Murakami A, Takahashi K: From tumor hypoxia to cancer progression: the implications of hypoxia-inducible factor- 1 expression in cancers. Anat Cell Biol, 2012; 45: 7378.

4) Nurwidya F, Takahashi F, Murakami A, Takahashi K: Epithelial mesenchymal transition in drug resistance and metastasis of lung cancer. Cancer Res Treat, 2012; 44: 151-156.

5) Takahashi K, Eves ND, Piper A, Song Y, Maher TM: Year in review 2011: acute lung injury, interstitial lung diseases, physiology, sleep and lung cancer. Respirology, 2012; 17: 554-562.

6) Togo S, Koike K, Seyama K: Cigarettesmoke induced emphysema in senescenceprone mice. The Senescence-Accelerated Mouse (SAM) : Achievements and Future Directions. 2012; Chapter 43: 475-488.

\section{Department of Metabolism and Endocrinology}

$<$ Original Articles >

* 1) Takeno K, Mita T, Nakayama S, Goto H, Komiya K, Abe H, Ikeda F, Shimizu T, Kanazawa A, Hirose T, Kawamori R,Watada H: Masked hypertension, endothelial dysfunction, and arterial stiffness in type 2 diabetes mellitus: a pilot study. Am J Hypertens, 2012; 25: $165-170$.

2) Katakami N, Kaneto H, Matsuoka T, Takahara M, Maeda N, Shimizu I, Ohno K, Osonoi T, Kawai K, Ishibashi F, Imamura K, Kashiwagi A, Kawamori R, Matsuhisa M, Funahashi T, Yamasaki Y, Shimomura I: Adiponectin G276T gene polymorphism is associated with cardiovascular disease in Japanese patients with type 2 diabetes. Atherosclerosis, 2012; 220: 437-442.

3) Nohara R, Daida H, Hata M, Kaku K, Kawamori R, Kishimoto J, Kurabayashi M, Masuda I, Sakuma I, Yamazaki T, Yokoi H, Yoshida M: Effect of intensive lipid-lowering therapy with rosuvastatin on progression of carotid intima-media thickness in Japanese patients: Justification for Atherosclerosis Regression Treatment (JART) study. Circ J, 2012; 76: 221-229.

4) Miyauchi K, Yamazaki T, Watada H, Tanaka Y, Kawamori R, Imai Y, Ikeda S, Kitagawa A, Ono Y, Murayama F, Choi JB, Suwa S, Hayashi D, Kishimoto J, Daida H: Management of home blood pressure by amlodipine combined with angiotensin II receptor blocker in type 2 diabetes. Circ J, 2012; 76: 21592166.

* 5) Yoshida M, Mita T, Yamamoto R, Shimizu T, Ikeda F, Ohmura C, Kanazawa A, Hirose T, Kawamori R, Watada H: Combination of the Framingham risk score and carotid intima-media thickness improves the prediction of cardiovascular events in patients with type 
2 diabetes. Diabetes Care, 2012; 35: 178-180.

6) Kawamori R, Inagaki N, Araki E, Watada H, Hayashi N, Horie Y, Sarashina A, Gong Y, von Eynatten M, Woerle HJ, Dugi KA: Linagliptin monotherapy provides superior glycaemic control versus placebo or voglibose with comparable safety in Japanese patients with type 2 diabetes: a randomized, placebo and active comparator-controlled, double-blind study. Diabetes Obes Metab, 2012; 14: 348357.

7) Freemantle N, Balkau B, Danchin N, Wang E, Marre M, Vespasiani G, Kawamori R, Home PD: Factors influencing initial choice of insulin therapy in a large international noninterventional study of people with type 2 diabetes. Diabetes Obes Metab, 2012; 14: 901-909.

8) Nakanishi R, Hirose T, Tamura Y, Fujitani Y, Watada H: Attempted suicide with liraglutide overdose did not induce hypoglycemia. Diabetes Res Clin Pract, 2012; 99: e3-e4.

9) Kawamori R, Tajima N, Iwamoto Y, Kashiwagi A, Shimamoto K, Kaku K, Sano H: Predictive values of serum insulin kinetics for reversion of impaired glucose tolerance to normal glucose tolerance and the effects of voglibose treatment: a retrospective post hoc analysis of a Japanese phase III study. Diabetology International, 2012; 3: 209-216.

10) Sato $Y$, Kondo K, Sone H, Kobayashi M, Kawamori R, Tamura Y, Atsumi Y, Oshida Y, Tanaka S, Suzuki S, Makita S, Ohsawa I, Imamura S: Present situation of exercise therapy for patients with diabetes mellitus in Japan: a nationwide survey. Diabetology International, 2012; 3: 86-91.

11) Tuohetimulati G, Uchida T, Toyofuku $Y$, Abe $\mathrm{H}$, Fujitani Y, Hirose T, Takeda S, Watada H: Effect of maternal high-fat diet on pancreatic beta cells of the offspring. Diabetology International, 2012; 3: 217-223.

12) Saito R, Yamada S, Yamamoto $Y$, Kodera T, Hara A, Tanaka Y, Kimura F, Takei I, Umezawa K, Kojima I: Conophylline suppresses pancreatic stellate cells and improves islet fibrosis in Goto-Kakizaki rats. Endocrinology, 2012; 153: 621-630.

13) Imamura M, Maeda $S$, Yamauchi $T$, Hara $K$,
Yasuda K, Morizono T, Takahashi A, Horikoshi M, Nakamura M, Fujita H, Tsunoda T, Kubo M, Watada H, Maegawa H, Okada-Iwabu M, Iwabu M, Shojima N, Ohshige T, Omori S, Iwata M, Hirose H, Kaku K, Ito C, Tanaka Y, Tobe K, Kashiwagi A, Kawamori R, Kasuga M, Kamatani N, Nakamura Y, Kadowaki T: A single-nucleotide polymorphism in ANK1 is associated with susceptibility to type 1 diabetes in Japanese populations. Hum Mol Genet, 2012; 21: 3042-3049.

14) Imanishi $Y$, Hashimoto J, Ando W, Kobayashi K, Ueda T, Nagata Y, Miyauchi A, Koyano H, Kaji H, Saito T, Oba K, Komatsu Y, Morioka T, Mori K, Miki T, Inaba M: Matrix extracellular phosphoglycoprotein is expressed in causative tumors of oncogenic osteomalacia. J Bone Miner Metab, 2012; 30: 93-99.

15) Kawamori R, Kaku K, Hanafusa T, Kashiwabara D, Kageyama S, Hotta N: Efficacy and safety of repaglinide vs nateglinide for treatment of Japanese patients with type 2 diabetes mellitus. J Diabetes Investig, 2012; 3: 302-308.

16) Maeda A, Golczak M, Chen Y, Okano K, Kohno H, Shiose S, Ishikawa K, Harte W, Palczewska G, Maeda T, Palczewski K: Primary amines protect against retinal degeneration in mouse models of retinopathies. Nat Chem Biol, 2012; 8: 170-178.

17) Fukuda $H$, Imamura $M$, Tanaka $Y$, Iwata $M$, Hirose H, Kaku K, Maegawa H, Watada H, Tobe K, Kashiwagi A, Kawamori R, Maeda S: A single nucleotide polymorphism within DUSP9 is associated with susceptibility to type 2 diabetes in a Japanese population. PLoS One, 2012; 7: e46263.

$<$ Reviews $>$

1) Klionsky DJ and 1269 others (Watada H) : Guidelines for the use and interpretation of assays for monitoring autophagy. Autophagy, 2012; 8: 445-544.

2) Mita T, Watada H: Glucagon like peptide-1 and atherosclerosis. Cardiovasc Hematol Agents Med Chem, 2012; 10: 309-318.

3) Liebl A, Prusty V, Valensi P, Kawamori R, Christiansen JS, Palmer AJ, Balschmidt P, Ligthelm R, Mohan V: Ten years of experience with biphasic insulin aspart 30: from 
drug development to the latest clinical findings. Drugs, 2012; 72: 1495-1520.

4) Sato J, Hirose T, Watada H: Continuous glucose monitoring system: Is it really accurate, safe and clinically useful? J Diabetes Investig, 2012; 3: 225-230.

\section{Department of Nephrology}

$<$ Original Articles $>$

1) Shimizu Y, Ishii A, Takahata A, Kajiyama $T$, Yamahatsu A, Io H, Kurusu A, Hamada C, Horikoshi S, Tomino Y: Campylobacter bacteremia in hemodialysis patients by eating raw meat-the importance of sanitary education. Case Rep Nephrol Urol, 2012; 2: 145151.

2) Wakabayashi K, Asanuma K, Takeda Y, Arakawa A, Ohsawa I, Horikoshi S, Yao T, Tomino Y: Hyaline vascular type of unicentric Castleman's disease with proliferation of glomerular endothelial cells. Clin Nephrol, 2012; Dec 4. [Epub ahead of print]

3) Sasaki Y, Shimizu Y, Nakata J, Kameda T, Muto M, Ohsawa I, Io H, Hamada C, Horikoshi S, Tomino Y: Thyroid storm masked by hemodialysis and glucocorticoid therapy in a patients with rheumatoid arthritis. Casa Rep Nephrol Urol, 2012; 2: 6-10.

4) Berthoux F, Suzuki H, Thibaudin L, Yanagawa H, Maillard N, Mariat C, Tomino Y, Julian BA, Novak J: Autoantibodies targeting galactose-deficient IgA1 associate with progression of IgA nephropathy. J Am Soc Nephrol, 2012; 23: 1579-1587.

5) Inuma J, Murakoshi M, Kobayashi T, Io H, Kaneko K, Takahashi T, Hamada C, Horikoshi S, Tomino Y: Relationship between acceleration plethysmography and aortic calcification index in chronic kidney disease patients. Hong Kong Journal of Nephrology, 2012; 14: 48-53.

6) Sekiguchi Y, Hamada C, Ro Y, Nakamoto H, Inaba M, Shimaoka T, Io H, Koyanagi I, Aruga S, Inuma J, Kaneko K, Hotta Y, Margetts PJ, Mochizuki H, Horikoshi S, Tomino Y: Differentiation of bone marrow-derived cells into regenerated mesothelial cells in peritoneal remodeling using a peritoneal fibrosis mouse model. J Artif Organs, 2012; 15: 272-282.
* 7) Sekiguchi Y, Zhang J, Patterson S, Liu L, Hamada C, Tomino Y, Margetts PJ: Rapamycin inhibits transforming growth factor $\beta$-induced peritoneal angiogenesis by blocking the secondary hypoxic response. J Cell Mol Med, 2012; 16: 1934-1945.

8) Ogasawara S, Hosojima M, Kaseda R, Kabasawa H, Yamamoto-Kabasawa K, Kurosawa H, Sato H, Iino N, Takeda T, Suzuki Y, Narita I, Yamagata K, Tomino Y, Gejyo F, Hirayama Y, Sekine S, Saito A: Significance of urinary full-length and ectodomain forms of megalin in patients with type 2 diabetes. Diabetes Care, 2012; 35: 1112-1118.

9) Takagi-Akiba M, Asanuma K, Tanida I, Tada N, Oliva Trejo JA, Nonaka K, Asanuma E, Kominami E, Ueno T, Tomino Y: Doxorubicin-induced glomerulosclerosis with proteinuria in GFP-GABARAP transgenic mice. Am J Physiol Renal Physiol, 2012; 302: F380F389.

10) Matsumoto M, Io H, Furukawa M, Okumura K, Masuda A, Seto T, Takagi M, Sato M, Nagahama L, Omote K, Hisada A, Horikoshi S, Tomino Y: Risk factors associated with increased left ventricular mass index in chronic kidney disease patients evaluated using echocardiography. J Nephrol, 2012; 25: 794-801.

11) Lydia A, Asanuma K, Nonaka K, Takagi M, Jeong KH, Kodama F, Asao R, Asanuma E, Prodjosudjadi W, Tomino Y: Effects of 22oxa-calcitriol on podocyte injury in adriamycin-induced nephrosis. Am J Nephrol, 2012; 35: $58-68$.

* 12) Kusaba G, Ohsawa I, Ishii M, Inoshita H, Takagi M, Tanifuji C, Takahashi K, Nakamoto J, Yoshida M, Ohi H, Horikoshi S, Kurihara $\mathrm{H}$, Tomino Y: Significance of broad distribution of electron-dense deposits in patients with IgA nephropathy. Med Mol Morphol, 2012; 45: 29-34.

13) Moch Sja'bani, Moch, Dharmeizar, Tomino Y: Mlati Study and Indonesia JigLoSemar Research and Care Centres/IJRC. NASKAH LENGKAP, YOGUYAKARTA, Feb 18, 2012; 41-55.

$\dagger$ 14) Alsagaff MY, Thaha M, Aminuddin M, Yogiarto RM, Yogiantoro M, Tomino Y: 
Asymmetric dimethylarginine: a novel cardiovascular risk factor in end-stage renal disease. J Int Med Res, 2012; 40: 340-349.

15) Ozaki S, Atsumi T, Hayashi T, Ishizu A, Kobayashi S, Kumagai S, Kurihara Y, Kurokawa MS, Makino H, Nagafuchi H, Nakabayashi K, Nishimoto N, Suka M, Tomino Y, Yamada H, Yamagata K, Yoshida M, Yumura W: Severity-based treatment for Japanese patients with MPO-ANCA-associated vasculitis: the JMAAV study. Mod Rheumatol, 2012; 22: 394-404.

* 16) Sato D, Suzuki Y, Kano T, Suzuki H, Matsuoka J, Yokoi H, Horikoshi S, Ikeda K, Tomino Y: Tonsillar TLR9 expression and efficacy of tonsillectomy with steroid pulse therapy in IgA nephropathy patients. Nephrol Dial Transplant, 2012; 27: 1090-1097.

17) Aoki $T$, Kaneko S, Tanimoto M, Gohda T, Hagiwara S, Murakoshi M, Ishikawa Y, Furukawa M, Funabiki K, Horikoshi S, Tomino Y: Identification of quzntitative trait loci for diabetic nephropathy in $\mathrm{KK}-\mathrm{Ay} / \mathrm{Ta}$ mice. J Nephrol, 2012; 25: 127-136.

18) Horikoshi S, Higurashi A, Kaneko E, Yoshimura H, Ohsawa I, Suzuki Y, Hamada C, Tomino Y: A new screening method for proteinuria using Erythrosin B an automated analyzer--rapid, sensitive and inexpensive determination. Clin Chim Acta, 2012; 413: 1087-1091.

19) Mugitani N, Shimizu Y, Satake K, Suzuki Y, Horikoshi S, Tomino Y: Acceleration of crescent formation by $\mathrm{L} 1$ retrotransposon in mouse BSA-induced Nephritis. J Nephrol, 2012; 26: 375-384.

20) Kawamura T, Joh K, Okonogi H, Koike K, Utsunomiya Y, Miyazaki Y, Matsushima M, Yoshimura M, Horikoshi S, Suzuki Y, Furusu A, Yasuda T, Shirai S, Shibata T, Endoh M, Hattori M, Akioka Y, Katafuchi R, Hashiguchi A, Kimura K, Matsuo S, Tomino Y; Study Group SI: A histologic classification of IgA nephropathy for predicting long-term prognosis: emphasis on end-stage renal disease. J Nephrol, doi: 10.5301/jn.5000151. Epub 2012 Jun 7.

*21) Asao R, Asanuma K, Kodama F, Akiba-Takagi M, Nagai-Hosoe Y, Seki T, Takeda Y,
Ohsawa I, Mano S, Matsuoka K, Kurosawa H, Ogasawara S, Hirayama Y, Sekine S, Horikoshi S, Hara M, Tomino Y: Relationships between levels of urinary podocalyxin, number of urinary podocytes, and histologic injury in adult patients with IgA nephropathy. Clin J Am Soc Nephrol, 2012; 7: 13851393.

22) Ohsawa I, Kusaba G, Ishii M, Sato N, Inoshita H, Onda K, Hashimoto A, Nagamachi S, Suzuki H, Shimamoto M, Ohi H, Horikoshi S, Tomino Y: Extraglomerular C3 deposition and metabolic impacts in patients with $\operatorname{IgA}$ nephropathy. Nephrol Dial Transplant, doi: 10.1093/ndt/gfs262. Epub 2012 Jul 6.

23) Okazaki K, Suzuki Y, Otsuji M, Suzuki H, Kihara M, Kajiyama T, Hashimoto A, Nishimura H, Brown R, Hall S, Novak J, Izui S, Hirose S, Tomino Y: Development of a model of early-onset IgA nephropathy. J Am Soc Nephrol, 2012; 23: 1364-1374.

24) Nakata J, Ohsawa I, Onda K, Tanimoto M, Kusaba G, Takeda Y, Kobayashi N, Asanuma K, Tanaka Y, Sato M, Inami Y, Suzuki H, Suzuki H, Masuda A, Nonaka K, Sasaki Y, Hisada A, Hamada C, Horikoshi S, Tomino Y: Risk of overestimation of kidney function using GFR-estimating equations in patients with low inulin clearance. J Clin Lab Anal, 2012; 26: 248-253.

25) Hara M, Yamagata K, Tomino Y, Saito A, Hirayama Y, Ogasawara S, Kurosawa H, Sekine S, Yan K: Urinary podocalyxin is an early marker for podocyte injury in patients with diabetes: establishment of a highly sensitive ELISA to detect urinary podocalyxin. Diabetologia, 2012; 55: 2913-2919.

26) Lei B, Nakano D, Fujisawa Y, Liu Y, Hitomi H, Kobori H, Mori H, Masaki T, Asanuma K, Tomino Y, Nishiyama A: N-type calcium channel inhibition with Cilnidipine elicits glomerular podocyte protection independent of sympathetic nerve inhibition. J Pharmacol Sci, 2012; 119: 359-367.

*27) Hashimoto A, Suzuki Y, Suzuki H, Ohsawa I, Brown R, Hall S, Tanaka Y, Novak J, Ohi H, Tomino Y: Determination of severity of murine IgA nephropathy by glomerular complement activation by aberrantly glycosylated 
IgA and immune complexes. Am J Pathol, 2012; 181: 1338-1347.

*28) Ishikawa Y, Gohda T, Tanimoto M, Omote K, Furukawa M, Yamaguchi S, Murakoshi M, Hagiwara S, Horikoshi S, Funabiki K, Tomino Y: Effect of exercise on kidney function, oxidative stress, and inflammation in type 2 diabetic KK-A (y) mice. Exp Diabetes Res, 2012; 2012: 702948.

29) Ishikawa $\mathrm{Y}$, Ito $\mathrm{T}$, Tanimoto $\mathrm{M}$, Hagiwara $\mathrm{S}$, Furukawa M, Yamaguchi S, Omote K, Asanuma K, Gohda T, Shimizu Y, Funabiki K, Horikoshi S, Tomino Y: Podocyte loss and albuminuria of KK-Ay mouse: A spontaneous animal model for human type 2 diabetic nephropathy. J Diabetes Mellitus, 2012; 2: 346-352.

* 30) Sato M, Io H, Tanimoto M, Shimizu Y, Fukui M, Hamada C, Horikoshi S, Tomino Y: Relationship between preoperative radial artery and postoperative arteriovenous fistula blood flow in hemodialysis patients. J Nephrol, 2012; 25: 726-731.

31) Aruga S, Hamada C, Inuma J, Hotta $Y$, Koyanagi I, Inaba M, Shimaoka T, Sekiguchi Y, Ro Y, Mano S, Io H, Kaneko K, Horikoshi S, Tomino Y: Patterns of matrix metalloproteinases and transforming growth factor-beta 1 expression during peritoneal repair in chlorhexidine induced peritoneal fibrosis mice. Hong Kong J Nephrol, 2012; 14: 7-16.

32) Okuda M, Horikoshi S, Matsumoto M, Tanimoto M, Yasui H, Tomino Y: Beneficial effect of Astragalus membranaceus on estimated glomerular filtration rate in patients with progressive chronic kidney disease. Hong Kong J Nephrol, 2012; 14: 17-23.

33) Kanda R, Hamada C, Kaneko K, Nakano T, Wakabayashi K, Io H, Horikoshi S, Tomino Y: Pentraxin 3 as a new biomarker of peritoneal injury in peritoneal dialysis patients. J Artif Organs, 2012; 16: 66-73.

*34) Okumura K, Io H, Matsumoto M, Seto T, Takagi M, Masuda A, Furukawa M, Nagahama L, Omote K, Hisada A, Hamada C, Horikoshi S, Tomino Y: Predictive factors associated with change rates of LV hypertrophy and renal dysfunction in CKD patients. Clin Nephrol, 2012; 79: 7-14.
35) Gohda T, Niewczas MA, Ficociello LH, Walker WH, Skupien J, Rosetti F, Cullere X, Johnson AC, Crabtree G, Smiles AM, Mayadas TN, Warram JH, Krolewski AS: Circulating TNF receptors 1 and 2 predict Stage 3 CKD in type 1 diabetes. J Am Soc Nephrol, 2012; 23: 516524.

36) Niewczas MA, Gohda T, Skupien J, Smiles AM, Walker WH, Rosetti F, Cullere X, Eckfeldt JH, Doria A, Mayadas TN, Warram JH, Krolewski AS: Circulating TNF receptors 1 and 2 predict ESRD in type 2 diabetes. J Am Soc Nephrol, 2012; 23: 507-515.

37) Gohda T, Walker WH, Wolkow P, Lee JE, Warram JH, Krolewski A, Niewczas MA: Elevated urinary excretion of immunoglobulins in non-proteinuric patients with type 1 diabetes. Am J Physiol Renal Physiol, 2012; 303: F157-F162.

38) Skupien J, Warram JH, Smiles AM, Niewczas MA, Gohda T, Pezzolesi MG, Cantarovich D, Stanton R, Krolewski AS: The early decline in renal function in patients with type 1 diabetes and proteinuria predicts the risk of end-stage renal disease. Kidney Int, 2012; 82: 589-597.

39) Iida H, Hasegawa T, Okuma K, Io H, Tomino Y, Ikeda S: Successfully maintained hemodialysis for the treatment of chronic renal failure in a patient with Hallopeau-Siemens type recessive dystrophic epidermolysis bullosa. J Dermatol, 2012; 39: 1088-1089.

40) Inoshita $H$, Ohsawa I, Onda K, Tamano $M$, Horikoshi S, Tomino Y: An analysis of functional activity via the three complement pathways during hemodialysis sessions: a new insight into the association between the lectin pathway and C5 activation. Clin Kidney J, 2012; 5: 401-404.

41) Horiuchi T, Ohi H, Ohsawa I, Fujita T, Matsushita M, Okada N, Seya T, Yamamoto T, Endo Y, Hatanaka M, Wakamiya N, Mizuno M, Nakao M, Okada H, Tsukamoto H, Matsumoto M, Inoue N, Nonaka M, Kinoshita T; Japanese Association for Complement Research: Guideline for hereditary angioedema (HAE) 2010 by the Japanese Association for Complement Research - secondary publication. Allergol Int, 2012; 61: 559-562. 
* 42) Masuda A, Hagiwara S, Tanimoto M, Kodama F, Okumura K, Nohara N, Matsumoto M, Maiguma M, Omote K, Io H, Kurusu A, Ohsawa I, Shimizu Y, Hamada C, Horikoshi S, Tomino Y: Effects of acetate-free citrate dialysate on glycoxidation and lipid peroxidation products in hemodialysis patients. Nephron Extra, 2012; 2: 256-268.

43) Otani M, Nakata J, Kihara M, Leroy V, Moll S, Wada Y, Izui S: O-glycosylated IgA rheumatoid factor induces IgA deposits and glomerulonephritis. J Am Soc Nephrol, 2012; 23: 438446.

44) Otani M, Kuroki A, Kikuchi S, Kihara M, Nakata J, Ito K, Furukawa J, Shinohara Y, Izui S: Sialylation determines the nephritogenicity of IgG3 cryoglobulins. J Am Soc Nephrol, 2012; 23: 1869-1878.

* 45) Nakata J, Suzuki Y, Suzuki H, Sato D, Kano T, Horikoshi S, Novak J, Tomino Y: Experimental evidence of cell dissemination playing a role in pathogenesis of IgA nephropathy in multiple lymphoid organs. Nephrol Dial Transplant, doi: 10.1093/ndt/gfs467. Epub 2012 Nov 6.

46) Leroy V, Kihara M, Baudino L, Brighouse G, Evans LH, Izui S: Sgp3 and TLR7 stimulation differentially alter the expression profile of modified polytropic retroviruses implicated in murine systemic lupus. J Autoimmun, 2012; 38: 361-368.

47) Kiryluk K, Li Y, Sanna-Cherchi S, Rohanizadegan M, Suzuki H, Eitner F, Snyder HJ, Choi M, Hou P, Scolari F, Izzi C, Gigante M, Gesualdo L, Savoldi S, Amoroso A, Cusi D, Zamboli P, Julian BA, Novak J, Wyatt RJ, Mucha K, Perola M, Kristiansson K, Viktorin A, Magnusson PK,
Thorleifsson G, Thorsteinsdottir U, Stefansson K, Boland A, Metzger M, Thibaudin L, Wanner C, Jager KJ, Goto S, Maixnerova D, Karnib HH, Nagy J, Panzer U, Xie J, Chen N, Tesar V, Narita I, Berthoux F, Floege J, Stengel B, Zhang H, Lifton RP, Gharavi AG: Geographic differences in genetic susceptibility to IgA nephropathy: GWAS replication study and geospatial risk analysis. PLoS Genet, 2012; 8: e1002765.

$\dagger 48$ ) Irijanto F, Rahajeng H, Kamajaya S, Djarwoto B, Sja'bani M, Sadewa AH, Shimizu Y, Tomino Y: High D allele frequency of ACE I/D gene polymorphism in familial hypertension in Javanese Indonesian. Juntendo Medical Journal, 2012; 58: 224-230.

*49) Saito K, Tomino Y: Usefulness of serum proteins as early markers of glomerular injury in patients with type 2 diabetic nephropathy. Juntendo Medical Journal, 2012; 58: 168-172.

$<$ Reviews $>$

1) Tomino Y: Leesons from the KK-Ay mouse, a spontaneous animal model for the treatment of human type 2 diabetic nephropathy. Nephrourol Mon, 2012; 4: 524-529.

2) Goda T, Tomino Y: Novel biomarkers for the progression of diabetic nephropathy: Soluble TNF receptors. Curr Diab Rep, 2012; 13: 560566.

3) Ohsawa I, Ishii M, Ohi H, Tomino Y: Pathological scenario with the mannose-binding lectin in patients with IgA nephropathy. J Biomed Biotechnol, 2012; 2012: 476739.

4) Klionsky DJ and 1269 others (Tomino Y) : Guidelines for the use and interpretation of assays for monitoring autophagy. Autophagy, 2012; 8: 445-544. 